\title{
Infecção hospitalar em pacientes cirúrgicos de hospital universitário ${ }^{1}$
}

\author{
Aldo da Cunha Medeiros ${ }^{2}$ \\ Tertuliano Aires Neto ${ }^{3}$ \\ Antônio Medeiros Dantas Filho ${ }^{3}$ \\ Francisco Edilson Leite Pinto $\mathrm{Jr}^{3}$ \\ Raquel Araújo Costa Uchôa ${ }^{4}$ \\ Mariana Rêgo de Carvalho ${ }^{4}$
}

\begin{abstract}
Medeiros AC, Aires Neto T, Dantas Filho AM, Pinto Jr FEL, Uchôa RAC, Carvalho MR. Infecção hospitalar em pacientes cirúrgicos de Hospital Universitário. Acta Cir Bras [online] 2003 vol 18 suppl 1. Disponível em www.scielo.br/acb.
\end{abstract}

RESUMO: Objetivo: Analisar fatores intercorrentes e a incidência da infecção em pacientes operados no Hospital Universitário da UFRN. Métodos: Foram estudados, através de protocolo previamente estabelecido, 3.120 pacientes internados que se submeteram a procedimentos cirúrgicos no período de janeiro de 1999 a outubro de 2002. Resultados: O índice de infecção hospitalar foi de 5,9\%, e a topografia de maior incidência foi a ferida operatória (3,7\%). Infecção respiratória ocorreu em $1,2 \%$, urinária em $0,6 \%$ e bacteremia em $0,1 \%$. O índice de infecção comunitária foi de $9,2 \%$, predominando infecção urinária (5\%) e respiratória $(2,1 \%)$. Quanto ao grau de contaminação das feridas operatórias, as feridas limpas (1479) apresentaram infecção em 2,9\%, as feridas limpascontaminadas (1277) em 6,0\% dos casos, as feridas contaminadas (270) em $15,1 \%$, e as ferida infectadas (94) resultaram em infecção em 30,75\% dos casos. Conclusão: Concluiu-se que a incidência de infecção cirúrgica foi compatível com os índices na literatura mundial. A partir desses dados, ratifica-se a importância de medidas de controle de infecção hospitalar de forma sistemática, como vem sendo realizado no hospital onde o estudo foi realizado.

DESCRITORES: Infecção hospitalar. Incidência. Pós-operatório. Cirurgia.

\section{INTRODUÇ̃̃O}

Apesar dos grandes avanços em todas as áreas da cirurgia, o controle da infecção continua sendo um grande desafio para a cirurgia. É cada vez mais atual a idéia de que a profilaxia é o maior aliado do cirurgião, fazendo-se necessário um grande esforço para manter a infecção sob controle dentro dos padrões aceitáveis e preconizados pelas instituições nacionais e internacionais que lidam com o problema.

De acordo com estatísticas oficiais norte-americanas, de 593.344 operações estudadas e realizadas entre
1986 e 1996, cerca de 15.523 (3\%) foram seguidas de infecção. Dois terços dessas infecções ocorreram próximas à incisão, enquanto outro terço envolveu órgãos ou espaços acessados durante as operações. Dos 551 pacientes infectados que morreram, $77 \%$ das mortes foram provocadas por infecções graves. A infecção cirúrgica portanto, segue considerada hoje o problema mais importante nas salas de operações ${ }^{1}$.

As infecções das feridas cirúrgicas continuam consumindo um percentual considerável dos recursos destinados à assistência à saúde. Embora a eliminação completa da infecção no paciente cirúrgico seja

1. Trabalho da Disciplina de Técnica Operatória e Cirurgia Experimental da UFRN.

2. Prof. Dr. do Departamento de Cirurgia-UFRN; Chefe do Núcleo de Cirurgia Experimental e da Disciplina de Técnica OperatóriaUFRN; Pesquisador nível 1 do CNPq.

3. Professor Adjunto de Técnica Operatória-UFRN; aluno do Programa de Pós-graduação (doutorado).

4. Aluna do Programa de Iniciação Científica. 
impossível, uma redução na sua incidência para um nível mínimo pode produzir grandes benefícios tanto em conforto para os pacientes quanto em recursos economizados. Para se discutir racionalmente as infecções em cirurgia, é necessário definir certos termos. As feridas cirúrgicas são classificadas em quatro categorias, de acordo com o seu grau de contaminação, conforme estabelecido pelo Colégio Americano de Cirurgiões ${ }^{2}$. Feridas limpas: têm reduzido potencial de infecção, não ocorre abertura de vísceras ocas ou infração da técnica asséptica; nas feridas limpas contaminadas ocorre abertura de víscera oca, com mínimo extravasamento de conteúdo ou pequenas infrações técnicas. As feridas contaminadas são decorrentes da abertura de víscera oca com grosseiro extravasamento de conteúdo, inflamação aguda sem pus, infrações grosseiras na técnica asséptica e lesões traumáticas com menos de seis horas. Finalmente, nas feridas sujas ou infectadas ocorre a presença de pus, víscera oca perfurada e lesões traumáticas com mais de seis horas de evolução.

A infecção em cirurgia é uma entidade clínica de múltiplos fatores envolvidos, e a necessidade de reduzir e controlar sua incidência determina a aplicação de medidas preventivas, educacionais e de controle epidemiológico que visam, através de um processo de conscientização coletiva, levar a taxas de infecção para limites aceitáveis para o tipo de clientela e de procedimentos realizados em cada hospital ${ }^{4}$.

O objetivo do presente trabalho foi verificar os índices de infecção e fatores intercorrentes nos pacientes operados no Hospital Universitário da UFRN, durante período pré-determinado.

\section{MÉTODOS}

Foram monitorizados, através de protocolo previamente estabelecido, 3.120 pacientes operados nas várias clínicas cirúrgicas do Hospital Universitário da UFRN, no período de janeiro de 1999 a outubro de 2002.

A vigilância foi realizada pela Comissão de Controle de Infecção Hospitalar para os casos cirúrgicos, com o método de coleta de dados baseado na busca ativa. As definições foram padronizadas conforme recomendação de publicações do Ministério da Saúde, e a metodologia utilizada foi aquela proposta pelo sistema NNISS, com modificações.

Ingressaram no protocolo todos os pacientes admitidos no período acima estabelecido, que se submeteram a procedimento cirúrgico. Os elementos necessários à elaboração dos índices de infecção foram registrados e condensados em planilha própria, em que foram considerados: caracterização de infecção comunitária e infecção hospitalar, topografia da infecção, procedimentos invasivos concomitantes, enquadramento das operações de acordo com o risco e grau de contaminação, incidência de infecção cirúrgica em relação ao grau de contaminação.

\section{RESULTADOS}

Dos 3.120 pacientes operados no período, 187 desenvolveram infecção hospitalar, correspondendo a uma taxa de infecção cirúrgica de 5,99\%. Infecções consideradas comunitárias, ou seja, aquelas que os pacientes já apresentavam antes do seu internamento e que não foram provocadas pelo ato operatório, foram detectadas em 289 pacientes, atingindo a incidência de $9,2 \%$. Nas infecções hospitalares, a maior incidência ocorreu na ferida operatória (3,7\%), seguida da infecção respiratória $(1,2 \%)$ e urinária $(0,6 \%)$. Quanto à infecção comunitária, a maior incidência ocorreu no tracto urinário $(6,2 \%)$ e em segundo lugar no respiratório (tabela 1).

Tabela 1 - Incidência de infecção hospitalar e comunitária de acordo com a topografia, em 3.120 pacientes operados.

\begin{tabular}{c|c|c}
\hline TOPOGRAFIA & HOSPITALAR & COMUNITÁRIA \\
\hline Respiratória & $37(1,2 \%)$ & $65(2,1 \%)$ \\
\hline Urinária & $19(0,6 \%)$ & $196(6,2 \%)$ \\
\hline Ferida operatória & $118(3,7 \%)$ & $12(0,4 \%)$ \\
\hline Intra-abdominal & $9(0,3 \%)$ & $9(0,3 \%)$ \\
\hline Bacteremia & $4(0,1 \%)$ & $7(0,2 \%)$ \\
\hline TOTAL & $187(5,99 \%)$ & $289(9,2 \%)$ \\
\hline
\end{tabular}

As operações classificadas como limpas foram realizadas em 1.479 pacientes. Nestes, ocorreu incidência de infecção hospitalar em 42 pacientes $(2,9 \%$ dos casos). Nos 1.277 pacientes submetidos a operações com feridas limpas-contaminadas, 76 apresentaram infecção de ferida operatória, significando um 
índice de infecção de $6 \%$. Quanto às 270 operações classificadas como contaminadas, $40(15,1 \%)$ pacientes apresentaram infecção e dos 94 casos de operações classificadas como sujas ou infectadas, $28(30,76 \%)$ apresentaram infecção da ferida cirúrgica (tabela 2).

Tabela 2 - Incidência de infecção cirúrgica de acordo com o potencial de contaminação das operações.

\begin{tabular}{c|c|c}
\hline CLASSIFICAÇÃO & No DE OPERAÇÕES & INCIDÊNCIA DE INFECÇÕES \\
\hline Limpas & $1479(47,4 \%)$ & $43(2,9 \%)$ \\
\hline Limpas contaminadas & $1277(40,92 \%)$ & $76(6,0 \%)$ \\
\hline Contaminadas & $270(8,64 \%)$ & $40(15,1 \%)$ \\
\hline Sujas ou infectadas & $94(3,01 \%)$ & $28(30,76 \%)$ \\
\hline TOTAL & $3120(100,0 \%)$ & $187(5,99 \%)$ \\
\hline
\end{tabular}

Os procedimentos invasivos aos quais os pacientes foram submetidos influenciaram os índices da infecção hospitalar, o que pôde ser claramente detectado quando foi relacionado o número de pacientes que usaram sonda vesical de demora $436(14 \%)$, cateter venoso central
$180(5,7 \%)$, e drenos cavitários $156(5 \%)$ entre outros dispositivos potenciais veiculadores de bactérias para o interior do organismo (tabela 3). A correlação entre uso de sonda vesical e infecção urinária foi mais marcante nos pacientes acometidos de infecção comunitária.

Tabela 3 - Procedimentos invasivos a que foram submetidos os pacientes operados.

\begin{tabular}{c|c}
\hline PROCEDIMENTOS & NÚMERO \\
\hline Sonda vesical & $436(14,0 \%)$ \\
\hline Cateter venoso central & $180(5,7 \%)$ \\
\hline Drenos cavitários & $156(5,0 \%)$ \\
\hline Traqueostomia & $29(0,92 \%)$ \\
\hline TOTAL & 621 \\
\hline
\end{tabular}

\section{DISCUSSÃO}

O Hospital Universitário da UFRN é um hospital terciário, um dos raros hospitais gerais públicos do Rio Grande do Norte, que atende toda a população do Estado e de parte da Paraíba. Sua clientela caracterizase por ser susceptível à infecção, pois é constituída de pacientes referenciados com carências nutricionais graves. Estudo realizado no mesmo hospital revelou que apenas $20 \%$ dos pacientes cirúrgicos pesquisados ingerem alimentos protéicos diariamente, $17,5 \%$ o fazem duas vezes por semana e $41,7 \%$ não se alimentam de proteínas ${ }^{3}$. O índice de operações limpas $(47,4 \%)$ observado nos operados foi aproximadamente igual ao detectado por Ferraz et $\mathrm{al}^{4}(41,9 \%)$, bem como outros dados de incidência observados, certamente pela semelhança entre as instituições que têm a mesma finalidade e oriundas da mesma região. Dos pacientes que se submeteram a este tipo de operação, 2,9\% desenvolveram infecção cirúrgica, índice satisfatório quando comparado aos índices obtidos por Ferraz et $a l^{4}$, Sawyer et $a l^{5}$, e Peter et $a l^{6}$, respectivamente $3,4 \%, 1,5 \%$ e $1,8 \%$. Quanto aos outros graus de contaminação da ferida operatória, os níveis de infecção observados estão em concordância com estudo realizado com 62.939 pacientes $^{5}$, no qual ocorreu incidência de 7,7\% de infecção em operações limpas-contaminadas, $15,2 \%$ em contaminadas e $40 \%$ em sujas. Os níveis observados no presente estudo foram $6,0 \%, 15,1 \% \mathrm{e}$ $30,7 \%$ respectivamente.

Considerando-se a topografia da infecção, o maior índice observado ocorreu na ferida operatória, coincidindo com os dados encontrados na literatura ${ }^{4,7}$, onde a infecção mais comum foi a da ferida operatória. $\mathrm{Na}$ presente casuística, esta apresentou-se com a incidência de $3,7 \%$ do total de operações realizadas, mas têm sido descritas incidências globais de $5,6 \% \%^{7}, 11 \%^{4}$. A incidência de infecção respiratória atingiu $1,2 \%$, urinária, $0,6 \%$ e infecção intracavitária $0,3 \%$. Alguns trabalhos publicados revelam incidências de $2,8^{4}$ e $3,2 \%{ }^{7}$ para infecção respiratória, urinária $2,8 \%{ }^{4}$ e $1,8 \%{ }^{7}$, compatíveis com as encontradas no presente trabalho. 
Culver et al ${ }^{9}$, monitorizando 84.691 pacientes operados em 44 hospitais americanos, de 1987 a 1990, encontraram taxas de infecção cirúrgica global de 3,7\%. No presente trabalho, foi verificada uma taxa de 5,99\%, compatível com níveis aceitáveis de infecção no doente cirúrgico, levando em consideração as condições nutricionais precárias em que os pacientes foram operados ${ }^{3}$.

Em relação aos procedimentos invasivos aos quais os pacientes foram submetidos, foi verificado um índice de infecção do trato urinário de $0,6 \%$ dos pacientes operados e $6,2 \%$ entre aqueles que tiveram infecção comunitária. Tal discrepância pode ser explicada pelo fato de que muitos pacientes portadores de hipertrofia prostática permanecem por tempo prolongado usando sonda de Foley aguardando o ato operatório e terminam adquirindo infecção que tem sido detectada por ocasião do internamento hospitalar. Levando-se em consideração o uso de catéter venoso central em 5,7\% do total de pacientes operados, foi observada a incidência de $0,1 \%$ de casos de bacteriemia provavelmente determinada pela presença desse fator invasivo. Tem sido relatado o índice global de $1 \%$ de sepse após o uso de todos os tipos de cateteres intravasculares ${ }^{8}$.

CONCLUSÃO: Os dados disponíveis no presente trabalho permitem concluir que a implantação do controle de infecção hospitalar de forma sistemática, à semelhança dos procedimentos institucionalizados e difundidos por Ferraz et $\mathrm{al}^{4,10}$, podem resultar em índices aceitáveis de infecção cirúrgica. Ao longo dos anos, a partir da implantação da Comissão de Controle de Infecção Hospitalar, com todas as sua atribuições, tem ocorrido maior controle no uso de antimicrobianos, procedimentos e rotinas, o que certamente tem contribuído para os resultados atingidos no Hospital Universitário-UFRN.

\section{REFERÊNCIAS}

1. Mitka M. Preventing surgical infection is more important than ever. JAMA 2000;283(1):44-45.

2. Altemeier WA, Burke JF, Pruitt Ba. Manual on control of infection in surgical patients of the American College of Surgeons. Philadelphia, J. P. Lippicott, 1976.

3. Medeiros AC, Dantas Jr JPD, Rodrigues JM, Ramos CCO, Brito CA. Deficiência na dieta e incidência do câncer do estômago. Rev Ass Med RN 1997; 3:1-4.

4. Ferraz EM, Ferraz AAB, Bacelar TS, D“Albuquerque HST, Vasconcedlos MDMM, Leão CS. Controle de infecção em cirurgia geral - resultado de um estudo prospectivo de 23 anos e 42.274 cirurgias. Rev Col Bras Cir 2001; 28: 17-26.

5. Sawyer RG, Pruett TL. Infecções das feridas. Cl Cir Am Norte 1994; 3 : 561-580

6. Peter JE, Cruse MB. Incidence of wound infection on the surgical services. Surg Cl North Am 1975; 55: 1269-1275.

7. Rebollo MH, Bernal JM, Llorca J, Rabasa JM, Revuelta JM. Nosocomial infections having cardiovascular operations: a multivariate analysis of risk factors. J Thorac Cardiovasc Surg 1996;112:908-13.

8. Garrison RN, Wilson MA. Infecções dos cateteres intra-venosos e centrais. Cl Cir Am Norte 1994; 3: 601-615.

9. Culver DH, Horan TC, Gaynes RP. Surgical wound infection rates by wound class, operative procedure, and risk index. Am J Med 1991; 91 (suppl 3B): 152s - 157s.

10. Ferraz EM. Incidência e custo das infecções; classificação das feridas segundo a etiologia e o risco de infecção. In: Ferraz EM, ed. Manual de controle de infecção em cirurgia do Colégio Brasileiro de Cirurgiões, São Paulo, Editora Pedagógica Universitária, 1982, pp 9-15.

Medeiros AC, Aires Neto T, Dantas Filho AM, Pinto Jr FEL, Uchôa RAC, Carvalho MR. Nosocomial infection in surgical patients of an Universitary Hospital. Acta Cir Bras [online] 2003 vol 18 suppl 1. Available in www.scielo.br/acb.

ABSTRACT: Objective: A study was accomplished in order to observe the infection incidence in patients operated in the Hospital Universitário-UFRN. Methods: a prospective study was performed involving 3120 patients submitted to surgical procedures from january 1999 to october 2000. Results: The nosocomial surgical infection ratio was $5,99 \%$. Surgical wound infection was the most common $(3,7 \%)$, followed by respiratory infection $(1,2 \%)$, urinary $(0,6 \%)$, intraabdominal $(0,3 \%)$ and bacteremia $(0,1 \%)$. The communitary infection ratio was $9,2 \%$. From these, the urynary infection was the most common $(5 \%)$, followed by respiratory $(2,1 \%)$. Infection occured in $2,9 \%$ of 1479 clean wounds, $6 \%$ of 1277 clean-contaminated, $15,1 \%$ of contaminated and $30,7 \%$ of infected wounds. Conclusion: These data permit to conclude that the incidence of surgical infection is compatible with that observed in the world literature. So, it confirms the importance of the nosocomial infection control in a systematic way, as it has been accomplished in the studied hospital.

KEY WORDS: Surgical infection. Postoperative. Incidence. Surgery.

\section{Correspondência:}

Prof. Dr. Aldo da Cunha Medeiros

Av. Miguel Alcides Araújo, 1889

Natal - RN - 59078-270 